\title{
Evolution of Lysine-Specific Demethylase 1 and REST Corepressor Gene Families and Their Molecular Interaction
}

\section{Montserrat Olivares}

Pontificia Universidad Católica de Chile

Gianluca Merello

Columbia University

\section{Daniel Verbel}

Pontificia Universidad Católica de Chile

\section{Marcela Gonzalez}

Pontificia Universidad Católica de Chile

\section{María Andrés}

Pontificia Universidad Católica de Chile

Juan Opazo ( $\square$ jopazo@gmail.com )

Austral University of Chile https://orcid.org/0000-0001-7938-4083

\section{Article}

Keywords: Lysine-specific demethylase 1A (LSD1), REST, gene families, molecular interaction, RCOR, Corepressor

Posted Date: December 3rd, 2021

DOI: https://doi.org/10.21203/rs.3.rs-1106901/v1

License: (9) This work is licensed under a Creative Commons Attribution 4.0 International License. Read Full License 


\title{
Evolution of Lysine-Specific Demethylase 1 and REST Corepressor gene families and their molecular interaction
}

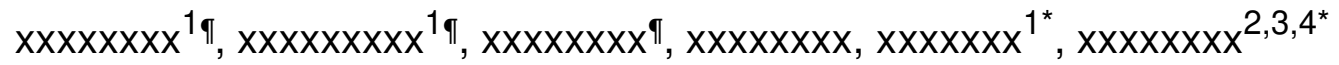

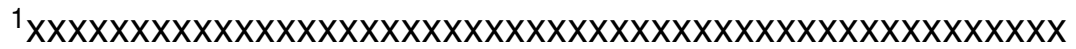

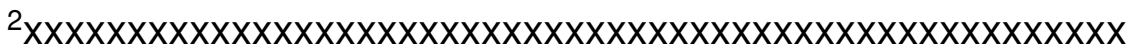

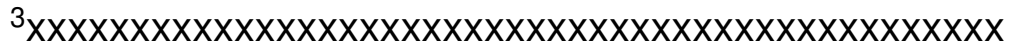 \\ ${ }^{4} X X X X X X X X X X X X X X X X X X X X X X X X X X X X X X X X X X X X X X X X X X X X X X X X X X X X X X X X X X X X$

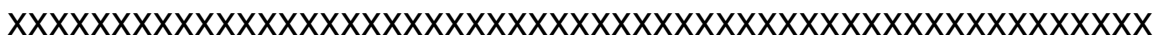 \\ I Equal contribution \\ * Correspondence to

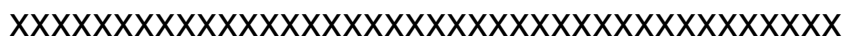

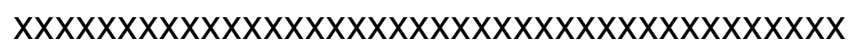




\section{Abstract}

Lysine-specific demethylase 1A (LSD1) binds to RCOR gene family of corepressors to erase transcriptionally active marks on histones. Functional diversity in these complexes depends on the type of RCOR included, which modulates the complex's catalytic activity. We studied the duplicative history of RCOR and LSD gene families, and analyzed the evolution of their interaction. We found that RCOR genes are the product of the two rounds of whole-genome duplications that occurred early in vertebrate evolution. In contrast, the origin of the LSD genes traces back before to the divergence of animals and plants. Coimmunoprecipitation experiments using resurrected RCOR and LSD1 proteins of the jawed vertebrate ancestor, and the common hop, date the origin of LSD1-RCOR interaction to the ancestor of animals, fungi, and plants. Overall, we trace LSD1-RCOR complex evolution and propose that animal, fungi, and plant non-model species offer advantages in addressing questions about the molecular biology of this epigenetic complex. 


\section{Introduction}

Lysine-specific demethylase $1 \mathrm{~A}$ (LSD1, KDM1A, BHC110, AOF2) is an epigenetic enzyme that represses gene expression by erasing transcriptionally permissive histone modifications (Shi et al. 2004; Forneris et al. 2005). LSD1 function and stability depend on forming a stable complex with a member of the RCOR family of transcriptional corepressors (Lee et al. 2005; Shi et al. 2005; Forneris et al. 2007). Mammalian LSD1 has a central SWIRM (Swi3p, Rsc8p, and Moira) domain and a C-terminus amino oxidase (AOD) catalytic domain. The AOD domain is interrupted by an alpha-helical tower domain of 92 amino acids that allows the interaction of LSD1 with the RCOR family of proteins, composed in mammals by RCOR1 (CoREST, CoREST1), RCOR2, and RCOR3 (Yang et al. 2006; Forneris et al. 2007; Á.P. Barrios et al. 2014). RCOR proteins share a characteristic structure, including three functional domains. An ELM2 (homology 2 of Egl27 and MTA1) domain, followed immediately by a SANT (Swi3, Ada2, N-Cor, and TFIIIB) domain. A second SANT domain localized at the $C$-terminus of RCOR proteins allows the interaction with nucleosomal DNA (Pilotto et al. 2015). The interSANT sequence or linker domain, sufficient for binding LSD1 (Shi et al. 2005), hugs the tower domain (Yang et al. 2006; A.P. Barrios et al. 2014).

Although the three RCORs interact with LSD1, the catalytic properties of the different complexes differ and functionally have been associated with different biological processes. For example, RCOR1 regulates differentiation into various cell lineages (Ballas et al. 2005; Yao et al. 2014; Xiong et al. 2020a) and represses the expression of viral genomes (Zhou et al. 2013), while RCOR2 maintains pluripotency and proliferation of embryonic stem cells (Yang et al. 2011). Although, both have relevant roles in central nervous system development (Wang et al. 2016; Monaghan et al. 2017). Unexpectedly, the RCOR3 study has lagged behind with no associated functions to date.

Regarding the evolution of these gene families, not much is known, although studies have reported distinct evolutionary patterns in plant and animal LSD proteins (Zhou and Ma 2008). Today, the availability of whole-genome sequences in a wide range of taxonomic groups opens an outstanding opportunity to shed light on the evolution of 
gene families. Understanding the duplicative history of gene families is required, among other things, to make biologically meaningful comparisons. Although several questions can be asked by analyzing the gene repertoire in representative species of a given taxonomic group, there are questions related to the LSDs and RCORs that require special attention. For example, when did the expansion of the RCOR repertoire happen? Are they the product of the whole-genome duplications or loci-specific duplications? What is the conservation pattern of the different domains of the LSD and RCOR genes? Is LSD1RCOR interaction restricted to metazoans?

In this work, we studied the evolutionary history of the LSD and RCOR gene families in animals. Our results suggest that RCOR genes are ohnologs and that their diversification occurred in the ancestor of jawed vertebrates, while the origin of LSD paralogs is much more ancient. According to their phyletic distribution, LSD1 and RCOR are widespread in metazoans, and are also found in fungi and plants. Our experiments resuscitating the RCOR and LSD1 proteins present in the ancestor of jawed vertebrates indicate that the LSD1-RCOR interaction precedes the RCOR repertoire expansion. Furthermore, our co-immunoprecipitation experiments using the common hop as a model system suggest that the origin of the LSD1-RCOR interaction traces back to the common ancestor of animals, fungi, and plants. 


\section{Results and discussion}

The RCOR gene repertoire expanded in the ancestor of jawed vertebrates.

To understand the duplicative history of the RCOR genes, we reconstructed gene phylogenies with different taxonomic samplings. The first analysis aimed to understand the evolution of RCOR genes in vertebrates (Fig. 1), whereas in the second, our sampling effort included representative species of all main groups of animals (Fig. 2).

In the first analysis, our maximum-likelihood tree recovered well supported clades corresponding to RCOR1 sequences from vertebrates and RCOR2, and RCOR3 sequences of jawed vertebrates (i.e., gnathostomes) (Fig. 1). In this tree, the clade containing RCOR1 sequences was recovered sister to RCOR3 clade; however, this relationship is not supported (Fig. 1). The clade containing RCOR2 sequences from jawed vertebrates was recovered sister to the RCOR1/RCOR3 clade (Fig. 1). Our analysis recovered a clade containing RCOR sequences from two jawless vertebrates (i.e., cyclostomes) species (inshore hagfish and sea lamprey) sister to the RCOR1 clade from jawed vertebrates (Fig. 1). This topology suggests different evolutionary scenarios: 1) the RCOR genes diversified in the vertebrate ancestor, and jawless vertebrates only retained one copy (RCOR1) or 2) the RCOR genes diversified in the ancestor of jawed vertebrates and the sister group relationship recovered in our gene tree is a phylogenetic artifact. This last scenario could be possible, given that resolving orthology between jawless and jawed vertebrates is a complex evolutionary problem because of the compositional biases of the former group (Qiu et al. 2011; Smith et al. 2013). Additionally, resolving phylogenetic relationships among vertebrates needs a taxonomic sampling that includes more than just vertebrate species.

To further understand if the RCOR genes diversified in the ancestor of vertebrates or jawed vertebrates, we performed a phylogenetic analysis extending our sampling to representative species of all main groups of animals and reducing the representation of vertebrates. In addition to showing the presence of a single copy gene in all major groups of animals other than vertebrates, our phylogenetic tree resolves the sister group relationship between jawless and jawed vertebrates (Fig. 2), and it is consistent with our second proposed scenario (Fig. 2). We recovered the monophyly of the vertebrate clade 
containing RCOR sequences with strong support (100/1/100, Fig. 2). The clade containing RCOR sequences from jawless vertebrates was recovered sister to the group containing the RCOR1, RCOR2, and RCOR3 clades from jawed vertebrates (Fig. 2), suggesting that the diversification of the RCOR genes occurred between 615 and 473 million years ago (Kumar et al. 2017) in the ancestor of jawed vertebrates, after the divergence from jawless vertebrates. Among jawed vertebrates, our gene tree recovered the sister group relationship between RCOR1 and RCOR2, while the RCOR3 clade was recovered sister to the RCOR1/RCOR2 clade (Fig. 2).

It is widely accepted that the evolution of vertebrates was shaped by ancient whole-genome duplications (WGDs) (Meyer and Schartl 1999; McLysaght et al. 2002; Dehal and Boore 2005; Hoegg and Meyer 2005; Putnam et al. 2008). The most accepted hypothesis invokes two rounds of WGD during the evolutionary history of vertebrates; however, the timing of these duplication events is still a matter of debate (Simakov et al. 2020; Nakatani et al. 2021). Genes that originated as results of WGDs are called ohnologues, in honor of Susumu Ohno, who was the first to propose the occurrence of two rounds of WGDs early in the evolution of vertebrates (Ohno 1970). The expansion of RCOR genes in jawed vertebrates suggests that they could be the result of the WGDs occurred during the evolution of vertebrates. After checking the repository of genes retained from WGDs in the vertebrate genomes (Singh and Isambert 2020), we confirmed that the RCOR genes are indeed the product of the vertebrate specific WGDs. Based on this evidence, we propose onhologs as the appropriate term to describe the homology relationship among them.

The role of each of the RCOR proteins is not yet clear. RCOR1 plays a specific role in keeping viral genomes in latency in neurons (Roizman et al. 2011). Studies in RCOR1 null mice reveal a crucial role of this protein in erythropoiesis and the proliferation of regulatory T cells (Yao et al. 2014; Xiong et al. 2020b). On the other hand, RCOR2 is significantly expressed in embryonic stem cells, regulating their proliferation and pluripotency (Yang et al. 2011). In the case of RCOR3, there is no evidence regarding its role in mammalian physiology and development. Together with other reports, this 
evidence highlights the contribution of WGDs to functional diversification among RCOR onhologs, supporting the pivotal role of WGDs in the origin of biological novelties.

\section{LSD1 and LSD2 were present in the ancestor of all animals}

Given the specificity of the interaction of RCOR proteins exclusively with LSD1 but not with its paralog Lysine-specific demethylase 1B (LSD2, KDM1B), we sought to investigate the evolutionary history of the LSD gene family in animals. To do so, we performed two phylogenetic analyses. In the first, we included representative species of the main groups of vertebrates (Fig. 3), whereas, in the second, our sampling effort expanded to the main groups of animals (Fig. S1).

In our first maximum likelihood tree, we recovered the monophyly of LSD1 and LSD2 genes from vertebrates (Fig. 3), suggesting that the ancestor of the group, which existed between 676 and 615 million years ago (Kumar et al. 2017), had both paralogs. To further understand the origin of these two genes, we analyzed their duplicative history now by including representative species of all main groups of animals. Our phylogenetic analyses also recovered the monophyly of each paralog, LSD1 and LSD2, suggesting that the ancestor of all animals had both paralogs in their genome (Fig. S1). Thus, our results are consistent with previous reports suggesting an early origin of LSD genes prior to the divergence of animals and plants (Zhou and Ma 2008).

\section{Phyletic distribution of RCOR and LSD genes}

To better describe the evolutionary history of RCOR and LSD genes in animals, we analyzed their phyletic distribution, i.e., their presence and absence in different animal groups. RCOR1, RCOR2, and RCOR3 are present in all major groups of vertebrates other than cyclostomes, which possess a single gene copy (named RCOR1/2/3) (Fig. 1, 2, and $4 \mathrm{~A}$ ). Interestingly, although RCOR1 and RCOR3 are present in all examined bird species, RCOR2 is restricted to species belonging to the orders Psittaciformes, Passeriformes, Accipitriformes, and Anseriformes (Table S1). A comparison of the chromosomal regions (flanked by NAA40 and MARK2 genes) in chicken and painted turtle confirms the absence of the RCOR2 gene in the chicken genome (Galliformes) (Fig. 4B). By contrast, in the New Caledonian crow (Passeriformes), conserved regions 
spanned all twelve exons of the RCOR2 gene (Fig. 4B). Given the phyletic distribution of RCOR2 in different orders of birds (Table S1), we suggest that the RCOR2 gene was lost independently in different bird lineages.

RCOR1 and RCOR2 play preponderant roles in the development of the cerebral cortex in mammals. Mice null for RCOR2 early in neural progenitor cells show decreased neocortex thickness and brain size (Wang et al. 2016), whereas knocking down RCOR1 in later stages of development alters the differentiation and migration of cortical neurons (Fuentes et al. 2012). Although there are no studies focused on the functional roles of RCORs in birds, it would be interesting to compare the formation of the dorsal telencephalon between birds with the full complement of RCOR genes with those lacking RCOR2.

In invertebrates, the RCOR gene is present in all analyzed phyla but in Tardigrada, Kinorhyncha, Loricifera, Nematomorpha, Sipuncula, Orthonectida, Micrognathozoa, Rhombozoa, Onychophora, Gnathostomulida, Gastrotricha, Entoprocta, Cycliophora, Chaentognatha, Acanthocephala, and Entoprocta (Table S2).

LSD1 and LSD2 genes are present in all main vertebrate groups (Fig. 3 and Table S3). However, LSD1 is not found in the invertebrates phyla Kinorhyncha, Loricifera, Nematomorpha, Sipuncula, Orthonectida, Micrognathozoa, Onychophora, Gnathostomulida, Gastrotricha, Entoprocta, Cycliophora, and Chaetognatha. LSD2, on the other hand, is absent in some Arthropods, as previously reported (Zhou and Ma 2008) and, Placozoa, Tardigrada, Kinorhyncha, Loricifera, Nematomorpha, Sipuncula, Rotifera, Platyhelminthes, Orthonectida, Micrognathozoa, Rhombozoa, Onychophora, Gnathostomulida, Gastrotricha, Entoprocta, Cycliophora, Chaetognatha, and Acanthocephala (Table S4).

Our analysis shows that there are more species where RCOR proteins co-exist with LSD1 than species with only one of them (Table S2 and S4). However, there are some exceptions. For instance, the water bear (Ramazzottius varieornatus) possesses LSD1 but not RCOR. Bearing in mind that identifying a gene does not necessarily mean that the protein is expressed, the water bear example would imply that LSD1 is functionally independent of RCOR in some animal lineages. This species and others could provide a suitable platform to investigate RCOR (or LSD1) functions that are LSD1 
(or RCOR)-independent to further understand the diverse range of biological processes in which these proteins participate.

\section{Mammals and turtles possess the regulatory sequences to express the LSD1-8a specifically in neurons}

The LSD1 gene possesses nineteen exons in humans, yet alternative splicing can incorporate two additional exons, generating four splice variants. Exon 2a (E2a) (60 bp) encodes for 20 amino acids, and microexon 8a (E8a) (12 bp) encodes for four aminoacids. Isoforms, including microexon E8a (LSD1-8a and LSD1 2a-8a), are exclusively expressed in neurons and are called neuronal LSD1 (neuroLSD1) (Zibetti et al. 2010). E8a microexon inclusion occurs during neuronal differentiation and enhances neurite morphogenesis in mammals (Zibetti et al. 2010). Further research has demonstrated a role for neuroLSD1 in modulating behavior (Rusconi et al. 2016; Wang et al. 2016). NeuroLSD1-KO mice display less anxiety in various behavioral tests (Rusconi et al. 2016), a diminished response to epileptogenic stimuli (Rusconi et al. 2016), and defects in spatial learning and memory (Wang et al. 2015; Longaretti et al. 2020). When discovered, neuroLSD1 was described as a mammalian-specific protein (Zibetti et al. 2010), although the same microexon was later identified in turtles (Wang et al. 2015), and an 8a-like exon was also described in zebrafish (Tamaoki et al. 2020).

Due to E8a's relevance in neuronal development and behavior, and the lack of a systematic study of the E8a phyletic distribution, we analyzed the presence of the E8a sequence at the LSD1 gene in representative species of all main groups of vertebrates. To annotate E8a sequences, we selected the intronic region between exons 8 and 9 , using the human LSD1 genomic sequence as reference. We considered as E8a, sequences aligning with human (Homo sapiens) E8a (Zibetti et al. 2010) or zebrafish (Danio rerio) E8a-like (Tamaoki et al. 2020), including conserved splice donor and acceptor sites (GT and AG, respectively). Our analyses confirmed that the E8a microexon (i.e., DTVK) is also present in turtles (Fig 4A), consistent with previous reports (Zibetti et al. 2010; Wang et al. 2015). On the other hand, crocodiles, amphibians, lizards, and snakes do not have the E8a sequence. Interestingly, the intronic region of these species is shorter than those in mammals and turtles (mean of $1537.5 \mathrm{bp}$ in crocodiles, 
amphibians, lizards, and snakes, whereas 9587.2 bp in mammals and turtles (Table S5). In birds, we found the E8a sequence only in species belonging to the orders of Phaethontiformes, Gaviiformes, Opisthocomiformes, and Accipitriformes (Fig. 4A and Table S5). We found a six amino acids coding sequence aligning with the E8a-like exon in bony fish, cartilaginous fish, and cyclostomes, previously described in zebrafish (Table S5) (Tamaoki et al. 2020). Thus, the microexon E8a appears to have been present in the vertebrate ancestor and lost multiple times as well as diverged (e.g., mammal vs. fish sequence) during the radiation of the group.

The E8a retention into LSD1 transcripts is regulated by three splicing factors (NOVA1, FUBP, and SRRM4). A palindromic sequence located $\sim 300$ bp towards the $3^{\prime}$ of E8a can trap the exon and its donor and acceptor splicing sites into a double-stranded RNA structure (Rusconi et al. 2015; Hwang et al. 2018). SRRM4 binds the UGCUGC motif upstream of the splice acceptor site of the exon E8a (Hwang et al. 2018), and together with NOVA1 and FUBP, they form a complex that can maintain a single-stranded pre-mRNA and therefore elicit exon E8a retention. We found that the palindromic sequence and the UGCUGC motif (from now, splicing regulatory sequences (SRS) are absent in most vertebrates, other than mammals and turtles (Fig. 4A). Given the presence of E8a and the absence of SRS in most vertebrate groups, we hypothesize that the expression of LSD-8a is not restricted to neuronal tissue in these animals. This finding agrees with previous results in zebrafish in which LSD1-8a is ubiquitously expressed (Tamaoki et al. 2020). On the other hand, LSD1-8a might be neuron-specific in turtles, although direct evidence for that is still lacking. If that is the case, though, turtles could serve as an interesting model to study the role of LSD1-8a in neuron development and plasticity.

\section{RCOR ohnologs linker and LSD1 tower domains display lower evolutionary conservation in comparison to their other functional domains}

LSD1 and RCOR have characteristic functional domains. The SWIRM and catalytic domains (AOD) in LSD1 and the interaction domains ELM2, SANT1 (for HDAC1/2), and SANT2 (for nucleosomal DNA and histone octamer) in RCORs. Additionally, LSD1- 
RCOR binding occurs between LSD1's tower domain and RCOR's inter-SANT or linker domain. Although all RCORs bind with similar affinity to LSD1, the resulting complexes differ in protein composition and catalytic capacities (Á.P. Barrios et al. 2014; Upadhyay et al. 2014; Pilotto et al. 2015), suggesting that expansion of the RCOR repertoire as a product of the whole-genome duplications that occurred early in the evolution of vertebrates have extended the functional capabilities of the LSD1-RCOR complexes.

To delve into the evolution of the RCOR and LSD1 molecular interaction, we studied the conservation of their functional domains in jawed vertebrates. To this end, multiple sequence alignments for each protein incorporating representative species of all major groups of jawed vertebrates (mammals, reptiles, birds, amphibians, bony fish, and cartilaginous fish) were performed. These alignments were used as an input for the ConSurf web server (Landau et al. 2005; Ashkenazy et al. 2010; Ashkenazy et al. 2016) to calculate positional conservation scores (Fig. 5). The highest mean residue conservation score (lowest value) was for the SANT1 domain followed by SANT2, ELM2, and lastly the linker domain (Fig. 5), although in RCOR2, the second SANT domain exhibited a mean conservation score similar to the ELM2 domain (RCOR1: ELM2 = $0.4364 \mid$ SANT1 $=-0.7590 \mid$ Linker $=-0.0090 \mid$ SANT2 $=-0.7149 ;$ RCOR2: ELM2 $=-$ $0.5151 \mid$ SANT1 $=-0.7079 \mid$ Linker $=0.2701 \mid$ SANT2 $=-0.4973 ;$ R3: ELM2 $=-0.2141 \mid$ SANT1 $=-0.6976 \mid$ Linker $=0.0349 \mid$ SANT2 $=-0.6092)$. In the case of LSD1, the $-\mathrm{N}$ and $-C$ portions of the AOD domain have the highest mean sequence conservation score. Unexpectedly, increased variability is observed in the tower domain (Fig. 5 and Fig. S2).

Low conservation of the linker domain in RCORs and tower domain in LSD1 was somewhat surprising given their role in forming the molecular complex. We analyzed the conservation of amino acids key to the interaction to get insight into this intriguing aspect. To this end, we carried out a comparative analysis between RCOR1 and RCOR3 based on their 3D structures in complex with LSD1 (Yang et al. 2006; Hwang et al. 2011; Á.P. Barrios et al. 2014). Using this information, we searched for the conservation of those key amino acids in equivalent positions in RCOR2. Interaction interfaces of LSD1-RCOR1 and LSD1-RCOR3 complexes were divided into four segments, I to IV (Fig. S2). In segment I, two salt bridges are formed between two Asp residues of RCOR1 and two Lys 
residues of LSD1. These residues are in the same position in RCOR3 forming equivalent salt bridges with LSD1. Segment II contains two salt bridges and one hydrogen bond between RCOR1 and LSD1. These are also conserved and in close contact in the LSD1RCOR3 complex. In segment III, a Lys in position 371 in RCOR3 substitutes the Arg in RCOR1 for the salt bridge with Asp495 of LSD1. Section IV is not essential for LSD1RCOR1 binding (Shi et al. 2005) but might aid LSD1s' indirect interaction with the DNA or the histone octamer (Kim et al. 2020). In segment IV, Lys397, Asp401, and Asp407 are adequately located to form two additional salt bridges between RCOR1-LSD1 and RCOR3-LSD1 (Fig. S2).

The residues mentioned above are either conserved or substituted by chemically equivalent amino acids in RCOR2, except for Asp320, which is replaced with a Gly (Fig. S2). High conservation of key amino acids in relevant positions for LSD1 interaction in the RCOR proteins (highlighted in orange in figure 5) indicates that they evolved before the repertoire expansion and have remained that way for the last 615 million years. On the other hand, we hypothesize that tower and linker domains variability has made possible the flexible encounter of different RCOR with LSD1 and the modulation of the catalytic activity that these exert on LSD1.

\section{Residues in most conserved positions in the primary structure of RCOR1 and RCOR3 linker domains constitute the interaction interface with LSD1}

Next, we expanded our analysis to all the residues that comprise the tower and linker domains (i.e., not only key residues for interaction). We investigated whether there is a relationship between the conservation score of a particular residue and its position in the 3D structure of the complex. Again, these analyses were restricted to RCOR1 and RCOR3, given their known 3D structure in complex with LSD1.

In the case of LSD1, 39.6\% of the residues of the tower domain were classified as highly conserved (Fig. 6A, dark red). Of this group, $54.7 \%$ of the amino acids directly participate in the interaction interface with RCOR1. These residues are distributed along the four segments analyzed in supplementary figure 3. The other group, i.e., the highly conserved amino acids that do not participate in the interaction interface, are located 
mostly on the basal/middle section of the Ta2 helix and the distal segment of the Ta1 helix (Fig. 6A). The functional significance of these amino acids remains to be studied.

As for RCOR $1,27.4 \%$ of residues in its linker domain displayed highly conserved scores (Fig. 6B). Remarkably, of this group of residues, $76.5 \%$ participate in the interaction interface with LSD1, and the majority are located in the La2 helix. Interestingly, three highly conserved residues in the La2 helix, Arg347, Gln350, and GIn354 (Fig. 6B, light blue arrows) are oriented towards the opposite direction of LSD1. The function of these amino acids is currently unknown. Finally, in the case of RCOR3, 26.6\% of the residues in its linker domain are highly conserved, and 62.5\% of them face LSD1 (Fig. $6 \mathrm{C})$. Furthermore, for RCOR3, highly conserved amino acids are not solely enriched in the L $\alpha 2$ helix, but are more evenly distributed between the La1 and La2 helices. In addition, and as seen with RCOR1, three La2 residues, Gln315, Asn316, and GIn319, oriented themselves on the opposite side of the L $\alpha 2$ helix, away from the interaction interface (Fig. 6C, light blue arrows).

Thus, this analysis sheds light on several conserved "blocks" that we hypothesize fulfill relevant molecular functions in each protein. As an example, Lys447 (Fig. 6A, red arrow) at LSD1s` tower domain, a highly conserved residue that does not participate in the interaction interface, has been previously shown to inform the structural relationships between LSD1 and HDAC1 within the RCOR1 ternary core complex (Song et al. 2020). Also, the most conserved amino acids in RCOR1 and RCOR3 linker domains were predictive of LSD1 interaction capabilities. Most interesting are the residues that proved highly conserved but whose molecular function has not yet been explored. Specifically, the outward-facing arginine, glutamine, and asparagine of RCOR1 and RCOR3 hold promise for further analyses.

\section{RCOR and LSD1 interaction precedes the RCOR repertoire expansion in the ancestor of jawed vertebrates}

Given the evolutionary pattern of repertoire expansion of RCOR genes in the jawed vertebrate ancestor and the lack of a systematic assessment of RCOR-LSD1 interaction in non-vertebrate species, we wondered if the single RCOR protein present in the jawed vertebrate ancestor, before the RCOR repertoire expansion, was able to interact with the 
corresponding LSD1 ancestral protein. To investigate this matter, we manually curated RCOR1, RCOR2, RCOR3, and LSD1 sequences to reconstruct the RCOR and LSD1 proteins present in the ancestor of jawed vertebrates. We then synthesized plasmids harboring RCOR and LSD1 attached to Myc and HA epitopes, respectively. We used these constructs for co-immunoprecipitation (Co-IP) assays on HEK293 cell lysates, with human RCOR1 and LSD1 sequences as controls (Fig. 7).

As expected, RCOR1 and LSD1 human proteins co-precipitated when we used anti-Myc antibodies for precipitation (Fig. 7C and S3). Importantly, we also found that the ancestral RCOR protein formed an immunocomplex with the ancestral LSD1 protein (Fig. $7 \mathrm{C}$ ), suggesting that both proteins already formed a complex in the jawed vertebrate ancestor, before the RCOR repertoire expansion, between 615 and 473 million years ago (Kumar et al. 2017). This result is in agreement with previous findings of an LSD1-RCOR protein complex in the fruit fly (Drosophila melanogaster) (Mačinković et al. 2019). Importantly, LSD1-RCOR interaction has only been demonstrated for human, rodent, and fruit fly proteins (Hakimi et al. 2003; Dallman et al. 2004; Shi et al. 2005; Yang et al. 2006; A.P. Barrios et al. 2014). The extent to which this interaction is also present in other animals or other non-animal species (e.g., fungi, plants) is currently unknown. This is partly due to the fact that the evolution of the RCOR gene family has remained unstudied to date.

\section{RCOR and LSD1 interaction is not restricted to animals}

As mentioned, LSD1-RCOR complex formation depends on linker-tower domain interaction. The tower domain is an interruption of LSD1`s AOD domain by a 90 amino acid protrusion composed of two intertwined alpha-helices. Tower domain presence/absence can be predicted by the size of the interrupting segment and its probability of formation of a coiled-coil structure (Lupas et al. 1991; Zhou and Ma 2008). Previous studies have shown that animal LSD1 shows a high probability of coiled-coil formation, suggesting that LSD1-RCOR interaction is widespread in metazoans (Lupas et al. 1991; Zhou and Ma 2008). Interestingly, Zhou and Ma 2008(Zhou and Ma 2008), described that although fungal LSDs' catalytic domain is interrupted, as in the case of animals, no coiled-coil structure is predicted. According to our search, however, the fly 
pathogenic fungus (Entomophthora muscae) possesses an LSD gene (GENC01027297.1) with a similar sized interruption and a predicted coiled-coil structure in its catalytic domain (Fig. 8A, B) (Lupas et al. 1991; Zhou and Ma 2008). To our surprise, this species also harbors an RCOR gene in its genome (Fig. 8A). This is in contrast to model organisms like the budding yeast (Saccharomyces cerevisiae) and the fission yeast (Schizosaccharomyces pombe). The budding yeast lacks an LSD gene, and the fission yeast has two LSD genes with no coiled-coil detected in their AOD domains and no RCOR identified in their genomes (Fig 8A) (Shi et al. 2004). Thus, RCOR proteins are not restricted to animals, and by virtue of their interacting domains, we hypothesize that LSD1-RCOR interaction is present at least in the fly pathogenic fungus (Entomophthora muscae).

To investigate if these interactions could be predicted in organisms that diverged previous to the animal/fungi ancestor, we turned our attention to plants. We chose plants because we identified an RCOR gene in the white myrtle (Rhodamnia Argentea, XP_030540128.1) in our initial searches for REST corepressor 1 genes. Further research on our part unveiled RCOR genes in the common hop (Humulus lupulus, LA342314.1) (Fig. 8A), centipedegrass (Eremochloa ophiuroides, GGKP01096563.1), narrowleaf lupin (Lupinus angustifolius, GGZP01006870.1), and sugar pine (Pinus lambertiana, GEUZ01007917.1), while RCOR was not found in the model organisms thale cress (Arabidopsis thaliana) and rice (Oryza sativa). Additionally, using molecular modeling, Martignago et al. 2019 (Martignago et al. 2019) suggested the presence of a tower domain in the LSD1 homolog of the moss Physcomitrella patens (Martignago et al. 2019). These findings suggest that LSD1-RCOR interaction could also be present in some species of plants. To investigate this, we used the sequences of the RCOR and LSD1 proteins of the common hop for additional Co-IP experiments. We chose the common hop because its LSD1 sequence is strongly predictive of a tower domain, as like the human LSD1, it has two consecutive coiled-coil motifs in its catalytic domain (Fig. 8A).

Co-IP experiments were conducted on lysates of HEK293 cultures previously transfected with plasmids encoding for common hop RCOR and LSD1. RCOR immunoprecipitation yielded coprecipitation of LSD1, suggesting that both proteins interact in the common hop. To our knowledge, this is the first evidence of LSD1-RCOR 
interaction using non-animal proteins. This finding suggests an evolutionary scenario in which the LSD1-RCOR interaction was already present in the animal/plant ancestor, implying that this molecular partnership is at least 1400 million years old. The fact that tower domain prediction (i.e., coiled-coil) was informative to suggest an interaction between these two plant proteins means that most probably, the molecular underpinnings of the association have remained largely conserved to date. This finding opens up an exciting front to studying the LSD1-RCOR epigenetic complex and highlights the utility of extending biological research to non-model organisms. Addressing questions like - to what chromatin domains is the plant LSD1-RCOR complex associated? What are the substrate/s and catalytic characteristics of this protein complex? How is LSD1 function regulated in plants? - can bring new knowledge that could be generalizable to their mammalian counterparts. This is especially relevant considering the emergence of LSD1 as a target to treat several types of cancers (Fang et al. 2019). Thus employing a comparative approach, i.e., taking advantage of other animals, fungal, and plant systems could improve our understanding of the structural and molecular biology of the LSD1RCOR complex with clear translational implications. 


\section{Acknowledgments}

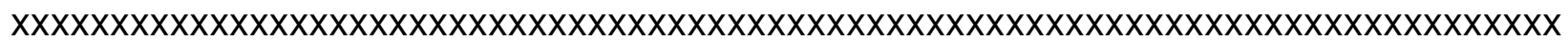

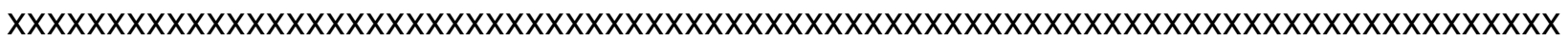

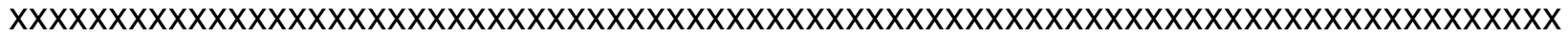

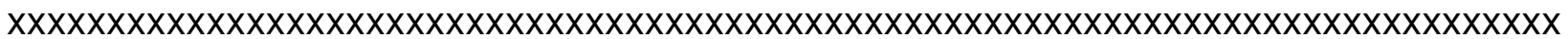
$X X X X X X X X X X X X X X X X X X X X X X X X X X X X X X X X X X X X X X$.

\section{Author contributions}

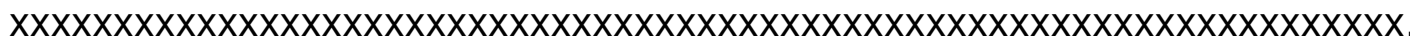

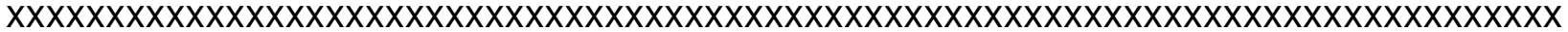

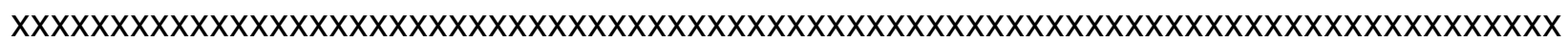
$X X X X X X X X X X X X X X X X X X X X X X X$

\section{Competing interests}

The authors declare no competing interests.

\section{Materials \& Correspondence.}

$x x x x x x x x x x x x x x x x x x x x x x x x x x x x$ 


\section{Figure legends}

Figure 1: Maximum likelihood tree showing relationships among $R C O R$ genes of vertebrates. Numbers on the nodes correspond to support from the ShimodairaHasegawa approximate likelihood-ratio test, approximate Bayes test, and ultrafast bootstrap values. The scale denotes substitutions per site and colors represent gene lineages. Mitotic deacetylase-associated SANT domain protein (MIDEAS) sequences from human (Homo sapiens), gorilla (Gorilla gorilla), mouse (Mus musculus), and ferret (Mustela putorius furo) were used as outgroup (not shown). For more details of the species included in the analysis, refer to table $\mathrm{S} 1$.

Figure 2: Maximum likelihood tree showing relationships among $R C O R$ genes of metazoa. Numbers on the nodes correspond to support from the Shimodaira-Hasegawa approximate likelihood-ratio test, approximate Bayes test, and ultrafast bootstrap values. The scale denotes substitutions per site and colors represent gene lineages. Mitotic deacetylase-associated SANT domain protein (MIDEAS) sequences from human (Homo sapiens), gorilla (Gorilla gorilla), mouse (Mus musculus), and ferret (Mustela putorius furo) were used as outgroup (not shown).

Figure 3: Maximum likelihood tree showing relationships among $L S D$ genes of vertebrates. Numbers on the nodes correspond to support from the ShimodairaHasegawa approximate likelihood-ratio test, approximate Bayes test, and ultrafast bootstrap values. The scale denotes substitutions per site and colors represent gene lineages. Monoamine-oxidases (MAO-A and MAO-B) sequences from human (Homo sapiens), chicken (Gallus gallus), spotted gar (Lepisosteus oculatus), and coelacanth (Latimeria chalumnae) were used as outgroup (not shown).

Figure 4: Phyletic distributions of RCOR and LSD genes, LSD1 microexon, and neuronspecific splicing regulatory sequences in vertebrates. A) Distribution of the RCOR and LSD genes, LSD1 microexon, and neuron-specific splicing regulatory sequences in main groups of vertebrates. B) Dot-plot of pairwise sequence similarity between the RCOR2 gene of the painted turtle (Chrysemys picta) and the corresponding syntenic region in the chicken (Gallus gallus) and New Caledonian crow (Corvus moneduloides). ${ }^{\text {RCOR2 }}$ is present only in the bird orders Psittaciformes, Passeriformes, Accipitriformes, and Anseriformes. 'Lampreys have a single copy of the RCOR gene. 'Some species of birds have a -DTVE- microexon.

Figure 5: LSD1 and RCORs normalized conservation scores of jawed vertebrates. Orange dots represent the conservation scores of the key amino acids for the LSD1RCORs interaction (Hwang et al. 2011). 
Figure 6: Color-coded 3D amino acid conservation of LSD1-RCOR protein complexes A) LSD1 tower domain residue conservation projected onto the structure of human LSD1RCOR1 (PDB: 2V1D). Red arrow shows Lys447 residue (see text). B) RCOR1 linkerSANT2 domain residue conservation projected onto the structure of human LSD1RCOR1 (PDB: 2V1D). Light blue arrows show Arg347, Gln350, and Gln354 residues (see text). C) RCOR3 linker-SANT2 domain residue conservation projected onto the structure of human LSD1-RCOR3 (PDB: 4CZZ). Light blue arrows show Gln315, Asn316, and Gln319 residues (see text).

Figure 7: Molecular interaction between the jawed vertebrate ancestral LSD1 and RCOR proteins. A) Graphical representation of the ancestral RCOR and LSD1 protein sequence reconstructions and co-immunoprecipitation experimental design. B) Multiple sequence alignments of the jawed vertebrate ancestral RCOR, human RCOR1, RCOR2, and RCOR3 linker and SANT2 domains. C) Sodium dodecyl sulphate-polyacrylamide gel electrophoresis (SDS-PAGE) followed by immunoblot detection of the immunoprecipitate obtained using an $\alpha-M y c$ antibody. Input represents whole-cell protein extracts, IgG serves as a negative control for the immunoprecipitation, while IP anti-Myc is the immunoprecipitation of ancestral RCOR protein. Molecular weights are indicated at right.

Figure 8: Molecular interaction between common hop LSD1 and RCOR proteins. A) (Left) Animal, fungi, and plant RCOR and LSD1 genes with their characteristic functional domains. The light brown section in LSD1's amino oxidase domain shows the prediction of a tower domain corresponding to an interruption in the amino oxidase domain and a strong prediction of a coiled-coil structure (Right). B) Multiple sequence alignment of human, Entomophthora muscae, common hop LSD1 and human LSD2 in the tower domain region of the human LSD1 sequence. C) Sodium dodecyl sulphatepolyacrylamide gel electrophoresis (SDS-PAGE) followed by immunoblot detection of the immunoprecipitate obtained using an a-Myc-RCOR antibody. Input represents whole-cell protein extracts, IgG serves as a negative control for the immunoprecipitation, while IP anti-Myc is the immunoprecipitation of common hop RCOR protein. Molecular weights are indicated at right. a LSD fission yeast homologs according to(Nicolas et al. 2006), b LSD thale cress homologs according to (Spedaletti et al. 2008).

Figure S1: Maximum likelihood tree phylogram showing relationships among $L S D$ genes of metazoa. Numbers on the nodes correspond to support from the ShimodairaHasegawa approximate likelihood-ratio test, approximate Bayes test, and ultrafast bootstrap values. The scale denotes substitutions per site and colors represent gene lineages. Monoamine-oxidases (MAO-A and MAO-B) sequences from human (Homo 
sapiens), chicken (Gallus gallus), spotted gar (Lepisosteus oculatus), and coelacanth (Latimeria chalumnae) were used as outgroup (not shown).

Figure S2: Comparison of the molecular details of the LSD1-RCOR1 and LSD1-RCOR3 interaction. A) (Top) Multiple sequence alignment of RCOR1, RCOR2, and RCOR3 linker domain. Highlighted positions represent RCOR interacting residues with LSD1 according to (Hwang et al. 2011). (Bottom) Structure and residue interactions of LSD1-RCOR1 and LSD1-RCOR3 at different interaction interfaces (numbered I-III) of tower-linker binding. B) (Top) Structure and residue interactions of LSD1-RCOR1 and LSD1-RCOR3 at interface IV. (Bottom) Multiple sequence alignment of RCOR1, RCOR2, and RCOR3 SANT2 domain. Highlighted positions represent RCOR interacting residues with LSD1. 


\section{Materials and Methods}

\section{Protein sequence collection and phylogenetic analyses}

We obtained lysine-specific demethylase 1 (LSD1), lysine-specific demethylase 2 (LSD2), REST Corepressor 1 (RCOR1), REST Corepressor 2 (RCOR2), and REST Corepressor 3 (RCOR3) protein sequences from the Ensembl v.102 (Yates et al. 2020) using orthology and paralogy estimates from the EnsemblCompara database(Herrero et al. 2016); these estimates were obtained from an automated pipeline that considers both synteny and phylogeny to generate orthology mappings. Further, we also retrieved sequences from the National Center for Biotechnology Information (NCBI) (Sharma et al. 2018) using the human (Homo sapiens) sequence as the reference for protein-BLAST (blastp) (Altschul et al. 1990) against the non-redundant database (nr) with default parameters. In each case, we corroborated the presence of all described protein domains (SWIRM/AOD for LSD, ELM2/SANT1/SANT2 for RCOR).

We implemented two types of analyses that involved different sampling strategies. The first analysis was aimed to understand the evolutionary history of these groups of genes in vertebrates, and our taxonomic sampling included representative species of all main groups of vertebrates. The second analysis was aimed to investigate the evolution of these genes in metazoans, thus our sampling included species of all main groups of animals. Accession numbers and details about the taxonomic sampling are available in Supplementary Table S1. We used the software MAFFT v.7 (Katoh and Standley 2013) to align amino acid sequences allowing the program to choose the alignment strategies (L-INS-i for LSD alignment in vertebrates and metazoa; FFT-NS-i for RCOR in vertebrates and metazoa). We estimate phylogenetic relationships using maximum likelihood (ML) approach as implemented in IQ-Tree v1.6.12 (Nguyen et al. 2015). We used the proposed model tool of IQ-Tree v.1.6.12 (Kalyaanamoorthy et al. 2017) to select the best-fitting models of amino acid substitution, which selected JTT+l+G4 for LSD in vertebrates and RCOR in vertebrates and metazoans. For LSD in metazoans, the model selected was $L G+F+R 10$. We assessed the node support using Shimodaira-Hasegawa approximate likelihood-ratio test (Guindon 2010), approximate Bayes test (Anisimova and Gascuel 2006; Guindon 2010), and ultrafast bootstrap approximation with 1000 pseudoreplicates (Minh et al. 2013; Hoang et al. 2018). We repeated each phylogenetic estimation ten times to explore the tree space, and the tree with the highest likelihood score was chosen. Sequences of monoamine-oxidases A and B (MAO-A and MAO-B), and Mitotic deacetylase-associated SANT domain protein (MIDEAS) were used as outgroup for LSD1/2 and RCOR1/2/3, respectively. 


\section{LSD1 alternative exon annotation}

To annotate the LSD1 alternative exon, called E-8a (Zibetti et al. 2010), in representative species of the main groups of vertebrates (Table S5), we retrieved LSD1 sequences from NCBI (Sharma et al. 2018) and Ensembl v.102 databases (Yates et al. 2020). We manually annotated the E-8a exon and regulatory sequences by comparing known sequences using the program Blast2seq v2.5 (Tatusova and Madden 1999) with default parameters using as reference the alternative exon previously described in human (Homo sapiens, CCDS53278.1), mouse (Mus musculus, XM_006539329.5), and zebrafish (Danio rerio, ENSDART00000085758.6) (Rusconi et al. 2015; Hwang et al. 2018; Tamaoki et al. 2020).

\section{Dot-plots}

We retrieved the chromosomal region containing the RCOR2 gene of the painted turtle (NW_007359864.1) and the corresponding syntenic region in the chicken (Chromosome 33-NC_008465.4), and New Caledonian crow (Chromosome 34- NC_045509.1) based

on the location of the flanking genes (NAA40 and MARK2). We aligned RCOR2 syntenic regions using PipMaker (Schwartz et al. 2000).

\section{Domain conservation analysis}

We performed four amino acid alignments corresponding to LSD1, RCOR1, RCOR2, and RCOR3, including representative species of all main groups of jawed vertebrates. Alignments were conducted using MAFFT v.7 (Katoh and Standley 2013), allowing the program to choose the alignment strategies (L-INS-i, in all cases). Then, using the alignments as input, we estimated normalized conservation scores for each alignment independently using the ConSurf WebServer (Landau et al. 2005; Ashkenazy et al. 2010; Ashkenazy et al. 2016). Protein domain positions were inferred using the human proteins as input using the InterPro web server (Blum et al. 2021).

\section{Ancestral sequence reconstructions}

We retrieved LSD1, LSD2, RCOR1, RCOR2, and RCOR3 coding sequences from NCBI (Sharma et al. 2018) using human (Homo sapiens), chicken (Gallus gallus), or zebrafish (Danio rerio) sequences as references for blastp searches (Altschul et al. 1990) against the non-redundant database (nr) with default parameters. Our taxonomic sampling included representative species of all main groups of chordates (mammals, birds, reptiles, bony fish, cartilaginous fish, cyclostomes, tunicates, and cephalochordates). Amino acid sequences were aligned using MAFFT v.7 (Katoh and Standley 2013) allowing the program to choose the alignment strategy (L-INS-i). Nucleotide alignments were 
generated using the amino acid alignments as templates using PAL2NAL (Suyama et al. 2006). Ancestral sequence reconstruction was performed using IQ-Tree v1.6.12 (Nguyen et al. 2015) (Table S.3), including an organismal tree based on the most updated phylogenetic hypotheses (Delsuc et al. 2008; Prum et al. 2015; Hara et al. 2018; Upham et al. 2019).

\section{Fungal and plant RCOR and LSD1 sequences}

Human RCOR1 and LSD1 protein sequences were used as reference for blastp searches using the non-redundant protein sequences (nr) database, or translated nucleotideBLAST (tblastn) (Gertz et al. 2006) using either nucleotide collection ( $\mathrm{nr} / \mathrm{nt}$ ) or transcriptome shotgun assembly (TSA) databases. Criteria to classify a product as RCOR were: e-value $<10^{-40}$ and presence of the four classical domains, ELM2, SANT1, linker, SANT2, in that order. Criteria to classify a protein as LSD1 were: e-value $<10^{-40}$, presence of the three classical domains, SWIRM, AOD-N, tower, AOD-C in that order. Tower prediction was based on the size of the interruption of the AOD domain ( $\sim 90$ residues) and a strong $(<0.8)$ prediction of coiled-coil structure formation in the center of the AOD domain. Coiled-coils were predicted using the webserver COILS (Lupas et al. 1991; Zhou and Ma 2008). RCOR and LSD1 functional domains were predicted using the Interpro web server (Blum et al. 2021).

\section{Co-immunoprecipitation assays}

The coding sequences for the jawed vertebrate ancestor LSD1 (from the start of the SWIRM domain until the end of the AOD domain), human LSD1 (same as above), and common hop LSD1 (same as above), and for the jawed vertebrate ancestor RCOR (from the start of the EML2 domain until the end of the SANT2 domain), human RCOR1 (same as above) and common hop RCOR (same as above), along with nuclear localization signals were obtained by synthetic synthesis (Twist Bioscience Corporation). Then, LSD1 sequences were cloned into the pCGN plasmid in frame with the HA epitope. RCOR sequences were cloned into the pCS2 + MT plasmid in frame with Myc epitopes. Frames were confirmed by sequencing and by western blot. Plasmid pairs were co-transfected in equimolar amounts into HEK293T cells. Twenty-four hours after transfection, whole RIPA extracts were immunoprecipitated with a-Myc antibodies as we have described previously (Hakimi et al. 2003; Dallman et al. 2004; Shi et al. 2005; Yang et al. 2006; A.P. Barrios et al. 2014). 


\section{References:}

Altschul SF, Gish W, Miller W, Myers EW, Lipman DJ. 1990. Basic local alignment search tool. J. Mol. Biol. 215:403-410.

Anisimova M, Gascuel O. 2006. Approximate likelihood-ratio test for branches: A fast, accurate, and powerful alternative. Syst. Biol. 55:539-552.

Ashkenazy H, Abadi S, Martz E, Chay O, Mayrose I, Pupko T, Ben-Tal N. 2016. ConSurf 2016: an improved methodology to estimate and visualize evolutionary conservation in macromolecules. Nucleic Acids Res. 44:W344-W350.

Ashkenazy H, Erez E, Martz E, Pupko T, Ben-Tal N. 2010. ConSurf 2010: calculating evolutionary conservation in sequence and structure of proteins and nucleic acids. Nucleic Acids Res. 38:W529W533.

Ballas N, Grunseich C, Lu DD, Speh JC, Mandel G. 2005. REST and its corepressors mediate plasticity of neuronal gene chromatin throughout neurogenesis. Cell 121:645-657.

Barrios ÁP, Gómez AV, Sáez JE, Ciossani G, Toffolo E, Battaglioli E, Mattevi A, Andrés ME. 2014. Differential properties of transcriptional complexes formed by the CoREST family. Mol. Cell. Biol. 34:2760-2770.

Barrios AP, Gomez AV, Saez JE, Ciossani G, Toffolo E, Battaglioli E, Mattevi A, Andres ME. 2014. Histone demethylase LSD1(KDM1A)-CoREST3 Complex. Available from:

http://dx.doi.org/10.2210/pdb4czz/pdb

Blum M, Chang H-Y, Chuguransky S, Grego T, Kandasaamy S, Mitchell A, Nuka G, Paysan-Lafosse T, Qureshi M, Raj S, et al. 2021. The InterPro protein families and domains database: 20 years on. Nucleic Acids Res. 49:D344-D354.

Dallman JE, Allopenna J, Bassett A, Travers A, Mandel G. 2004. A conserved role but different partners for the transcriptional corepressor CoREST in fly and mammalian nervous system formation. $J$. Neurosci. 24:7186-7193.

Dehal P, Boore JL. 2005. Two rounds of whole genome duplication in the ancestral vertebrate. PLoS Biol. 3:e314.

Delsuc F, Tsagkogeorga G, Lartillot N, Philippe H. 2008. Additional molecular support for the new chordate phylogeny. Genesis 46:592-604.

Fang Y, Liao G, Yu B. 2019. LSD1/KDM1A inhibitors in clinical trials: advances and prospects. J. Hematol. Oncol. 12:129.

Forneris F, Binda C, Adamo A, Battaglioli E, Mattevi A. 2007. Structural basis of LSD1-CoREST selectivity in histone H3 recognition. J. Biol. Chem. 282:20070-20074.

Forneris F, Binda C, Vanoni MA, Battaglioli E, Mattevi A. 2005. Human histone demethylase LSD1 reads the histone code. J. Biol. Chem. 280:41360-41365.

Fuentes P, Cánovas J, Berndt FA, Noctor SC, Kukuljan M. 2012. CoREST/LSD1 control the development of pyramidal cortical neurons. Cereb. Cortex 22:1431-1441.

Gertz EM, Yu Y-K, Agarwala R, Schäffer AA, Altschul SF. 2006. Composition-based statistics and 
translated nucleotide searches: improving the TBLASTN module of BLAST. BMC Biol. 4:41.

Guindon S. 2010. Bayesian estimation of divergence times from large sequence alignments. Mol. Biol. Evol. 27:1768-1781.

Hakimi M-A, Dong Y, Lane WS, Speicher DW, Shiekhattar R. 2003. A candidate X-linked mental retardation gene is a component of a new family of histone deacetylase-containing complexes. $J$. Biol. Chem. 278:7234-7239.

Hara Y, Yamaguchi K, Onimaru K, Kadota M, Koyanagi M, Keeley SD, Tatsumi K, Tanaka K, Motone F, Kageyama Y, et al. 2018. Shark genomes provide insights into elasmobranch evolution and the origin of vertebrates. Nat Ecol Evol 2:1761-1771.

Herrero J, Muffato M, Beal K, Fitzgerald S, Gordon L, Pignatelli M, Vilella AJ, Searle SMJ, Amode R, Brent S, et al. 2016. Ensembl comparative genomics resources. Database [Internet] 2016:bav096. Available from: http://dx.doi.org/10.1093/database/bav096

Hoang DT, Chernomor O, von Haeseler A, Minh BQ, Vinh LS. 2018. UFBoot2: Improving the Ultrafast Bootstrap Approximation. Mol. Biol. Evol. 35:518-522.

Hoegg S, Meyer A. 2005. Hox clusters as models for vertebrate genome evolution. Trends Genet. 21:421-424.

Hwang I, Cao D, Na Y, Kim D-Y, Zhang T, Yao J, Oh H, Hu J, Zheng H, Yao Y, et al. 2018. Far Upstream Element-Binding Protein 1 Regulates LSD1 Alternative Splicing to Promote Terminal Differentiation of Neural Progenitors. Stem Cell Reports 10:1208-1221.

Hwang S, Schmitt AA, Luteran AE, Toone EJ, McCafferty DG. 2011. Thermodynamic characterization of the binding interaction between the histone demethylase LSD1/KDM1 and CoREST. Biochemistry 50:546-557.

Kalyaanamoorthy S, Minh BQ, Wong TKF, von Haeseler A, Jermiin LS. 2017. ModelFinder: fast model selection for accurate phylogenetic estimates. Nat. Methods 14:587-589.

Katoh K, Standley DM. 2013. MAFFT multiple sequence alignment software version 7: improvements in performance and usability. Mol. Biol. Evol. 30:772-780.

Kim S-A, Zhu J, Yennawar N, Eek P, Tan S. 2020. Crystal Structure of the LSD1/CoREST Histone Demethylase Bound to Its Nucleosome Substrate. Mol. Cell 78:903-914.e4.

Kumar S, Stecher G, Suleski M, Hedges SB. 2017. TimeTree: A Resource for Timelines, Timetrees, and Divergence Times. Mol. Biol. Evol. 34:1812-1819.

Landau M, Mayrose I, Rosenberg Y, Glaser F, Martz E, Pupko T, Ben-Tal N. 2005. ConSurf 2005: the projection of evolutionary conservation scores of residues on protein structures. Nucleic Acids Research [Internet] 33:W299-W302. Available from: http://dx.doi.org/10.1093/nar/gki370

Lee MG, Wynder C, Cooch N, Shiekhattar R. 2005. An essential role for CoREST in nucleosomal histone 3 lysine 4 demethylation. Nature 437:432-435.

Longaretti A, Forastieri C, Toffolo E, Caffino L, Locarno A, Misevičiūtè I, Marchesi E, Battistin M, Ponzoni L, Madaschi L, et al. 2020. LSD1 is an environmental stress-sensitive negative modulator of the glutamatergic synapse. Neurobiol Stress 13:100280.

Lupas A, Van Dyke M, Stock J. 1991. Predicting coiled coils from protein sequences. Science 252:11621164. 
Mačinković I, Theofel I, Hundertmark T, Kovač K, Awe S, Lenz J, Forné I, Lamp B, Nist A, Imhof A, et al. 2019. Distinct CoREST complexes act in a cell-type-specific manner. Nucleic Acids Res. 47:1164911666.

Martignago D, Bernardini B, Polticelli F, Salvi D, Cona A, Angelini R, Tavladoraki P. 2019. The Four FADDependent Histone Demethylases of Arabidopsis Are Differently Involved in the Control of Flowering Time. Front. Plant Sci. 10:669.

McLysaght A, Hokamp K, Wolfe KH. 2002. Extensive genomic duplication during early chordate evolution. Nat. Genet. 31:200-204.

Meyer A, Schartl M. 1999. Gene and genome duplications in vertebrates: the one-to-four (-to-eight in fish) rule and the evolution of novel gene functions. Curr. Opin. Cell Biol. 11:699-704.

Minh BQ, Nguyen MAT, von Haeseler A. 2013. Ultrafast approximation for phylogenetic bootstrap. Mol. Biol. Evol. 30:1188-1195.

Monaghan CE, Nechiporuk T, Jeng S, McWeeney SK, Wang J, Rosenfeld MG, Mandel G. 2017. REST corepressors RCOR1 and RCOR2 and the repressor INSM1 regulate the proliferation-differentiation balance in the developing brain. Proc. Natl. Acad. Sci. U. S. A. 114:E406-E415.

Nakatani Y, Shingate P, Ravi V, Pillai NE, Prasad A, McLysaght A, Venkatesh B. 2021. Reconstruction of proto-vertebrate, proto-cyclostome and proto-gnathostome genomes provides new insights into early vertebrate evolution. Nat. Commun. 12:4489.

Nguyen L-T, Schmidt HA, von Haeseler A, Minh BQ. 2015. IQ-TREE: a fast and effective stochastic algorithm for estimating maximum-likelihood phylogenies. Mol. Biol. Evol. 32:268-274.

Nicolas E, Lee MG, Hakimi M-A, Cam HP, Grewal SIS, Shiekhattar R. 2006. Fission yeast homologs of human histone $\mathrm{H} 3$ lysine 4 demethylase regulate a common set of genes with diverse functions. $J$. Biol. Chem. 281:35983-35988.

Ohno S. 1970. Evolution by Gene Duplication. Springer Berlin Heidelberg

Pilotto S, Speranzini V, Tortorici M, Durand D, Fish A, Valente S, Forneris F, Mai A, Sixma TK, Vachette $\mathrm{P}$, et al. 2015. Interplay among nucleosomal DNA, histone tails, and corepressor CoREST underlies LSD1-mediated H3 demethylation. Proc. Natl. Acad. Sci. U. S. A. 112:2752-2757.

Prum RO, Berv JS, Dornburg A, Field DJ, Townsend JP, Lemmon EM, Lemmon AR. 2015. A comprehensive phylogeny of birds (Aves) using targeted next-generation DNA sequencing. Nature 526:569-573.

Putnam NH, Butts T, Ferrier DEK, Furlong RF, Hellsten U, Kawashima T, Robinson-Rechavi M, Shoguchi E, Terry A, Yu J-K, et al. 2008. The amphioxus genome and the evolution of the chordate karyotype. Nature 453:1064-1071.

Qiu H, Hildebrand F, Kuraku S, Meyer A. 2011. Unresolved orthology and peculiar coding sequence properties of lamprey genes: the KCNA gene family as test case. BMC Genomics 12:325.

Roizman B, Zhou G, Du T. 2011. Checkpoints in productive and latent infections with herpes simplex virus 1: conceptualization of the issues. J. Neurovirol. 17:512-517.

Rusconi F, Grillo B, Ponzoni L, Bassani S, Toffolo E, Paganini L, Mallei A, Braida D, Passafaro M, Popoli $\mathrm{M}$, et al. 2016. LSD1 modulates stress-evoked transcription of immediate early genes and emotional behavior. Proc. Natl. Acad. Sci. U. S. A. 113:3651-3656.

Rusconi F, Paganini L, Braida D, Ponzoni L, Toffolo E, Maroli A, Landsberger N, Bedogni F, Turco E, 
Pattini L, et al. 2015. LSD1 Neurospecific Alternative Splicing Controls Neuronal Excitability in Mouse Models of Epilepsy. Cereb. Cortex 25:2729-2740.

Schwartz S, Zhang Z, Frazer KA, Smit A, Riemer C, Bouck J, Gibbs R, Hardison R, Miller W. 2000. PipMaker--a web server for aligning two genomic DNA sequences. Genome Res. 10:577-586.

Sharma S, Ciufo S, Starchenko E, Darji D, Chlumsky L, Karsch-Mizrachi I, Schoch CL. 2018. The NCBI BioCollections Database. Database [Internet] 2018. Available from: http://dx.doi.org/10.1093/database/bay006

Shi Y-J, Matson C, Lan F, Iwase S, Baba T, Shi Y. 2005. Regulation of LSD1 histone demethylase activity by its associated factors. Mol. Cell 19:857-864.

Shi Y, Lan F, Matson C, Mulligan P, Whetstine JR, Cole PA, Casero RA, Shi Y. 2004. Histone demethylation mediated by the nuclear amine oxidase homolog LSD1. Cell 119:941-953.

Simakov O, Marlétaz F, Yue J-X, O'Connell B, Jenkins J, Brandt A, Calef R, Tung C-H, Huang T-K, Schmutz J, et al. 2020. Deeply conserved synteny resolves early events in vertebrate evolution. Nat Ecol Evol 4:820-830.

Singh PP, Isambert H. 2020. OHNOLOGS v2: a comprehensive resource for the genes retained from whole genome duplication in vertebrates. Nucleic Acids Res. 48:D724-D730.

Smith JJ, Kuraku S, Holt C, Sauka-Spengler T, Jiang N, Campbell MS, Yandell MD, Manousaki T, Meyer A, Bloom OE, et al. 2013. Sequencing of the sea lamprey (Petromyzon marinus) genome provides insights into vertebrate evolution. Nat. Genet. 45:415-421, 421e1-e2.

Song Y, Dagil L, Fairall L, Robertson N, Wu M, Ragan TJ, Savva CG, Saleh A, Morone N, Kunze MBA, et al. 2020. Mechanism of Crosstalk between the LSD1 Demethylase and HDAC1 Deacetylase in the CoREST Complex. Cell Rep. 30:2699-2711.e8.

Spedaletti V, Polticelli F, Capodaglio V, Schininà ME, Stano P, Federico R, Tavladoraki P. 2008. Characterization of a lysine-specific histone demethylase from Arabidopsis thaliana. Biochemistry 47:4936-4947.

Suyama M, Torrents D, Bork P. 2006. PAL2NAL: robust conversion of protein sequence alignments into the corresponding codon alignments. Nucleic Acids Res. 34:W609-W612.

Tamaoki J, Takeuchi M, Abe R, Kaneko H, Wada T, Hino S, Nakao M, Furukawa Y, Kobayashi M. 2020. Splicing- and demethylase-independent functions of LSD1 in zebrafish primitive hematopoiesis. Sci. Rep. 10:8521.

Tatusova TA, Madden TL. 1999. BLAST 2 Sequences, a new tool for comparing protein and nucleotide sequences. FEMS Microbiol. Lett. 174:247-250.

Upadhyay G, Chowdhury AH, Vaidyanathan B, Kim D, Saleque S. 2014. Antagonistic actions of Rcor proteins regulate LSD1 activity and cellular differentiation. Proc. Natl. Acad. Sci. U. S. A. 111:80718076.

Upham NS, Esselstyn JA, Jetz W. 2019. Inferring the mammal tree: Species-level sets of phylogenies for questions in ecology, evolution, and conservation. PLoS Biol. 17:e3000494.

Wang J, Telese F, Tan Y, Li W, Jin C, He X, Basnet H, Ma Q, Merkurjev D, Zhu X, et al. 2015. LSD1n is an $\mathrm{H} 4 \mathrm{~K} 20$ demethylase regulating memory formation via transcriptional elongation control. Nat. Neurosci. 18:1256-1264.

Wang Y, Wu Q, Yang P, Wang C, Liu J, Ding W, Liu W, Bai Y, Yang Y, Wang H, et al. 2016. LSD1 co- 
repressor Rcor2 orchestrates neurogenesis in the developing mouse brain. Nat. Commun. 7:10481.

Xiong Y, Wang L, Di Giorgio E, Akimova T, Beier UH, Han R, Trevisanut M, Kalin JH, Cole PA, Hancock WW. 2020a. Inhibiting the coregulator CoREST impairs Foxp3 Treg function and promotes antitumor immunity. Journal of Clinical Investigation [Internet] 130:1830-1842. Available from:

http://dx.doi.org/10.1172/jci131375

Xiong Y, Wang L, Di Giorgio E, Akimova T, Beier UH, Han R, Trevisanut M, Kalin JH, Cole PA, Hancock WW. 2020b. Inhibiting the coregulator CoREST impairs Foxp3+ Treg function and promotes antitumor immunity. J. Clin. Invest. 130:1830-1842.

Yang M, Gocke CB, Luo X, Borek D, Tomchick DR, Machius M, Otwinowski Z, Yu H. 2006. Structural basis for CoREST-dependent demethylation of nucleosomes by the human LSD1 histone demethylase. Mol. Cell 23:377-387.

Yang P, Wang Y, Chen J, Li H, Kang L, Zhang Y, Chen S, Zhu B, Gao S. 2011. RCOR2 is a subunit of the LSD1 complex that regulates ESC property and substitutes for SOX2 in reprogramming somatic cells to pluripotency. Stem Cells 29:791-801.

Yao H, Goldman DC, Nechiporuk T, Kawane S, McWeeney SK, Tyner JW, Fan G, Kerenyi MA, Orkin SH, Fleming WH, et al. 2014. Corepressor Rcor1 is essential for murine erythropoiesis. Blood 123:31753184.

Yates AD, Achuthan P, Akanni W, Allen J, Allen J, Alvarez-Jarreta J, Amode MR, Armean IM, Azov AG, Bennett R, et al. 2020. Ensembl 2020. Nucleic Acids Res. 48:D682-D688.

Zhou G, Du T, Roizman B. 2013. HSV carrying WT REST establishes latency but reactivates only if the synthesis of REST is suppressed. Proc. Natl. Acad. Sci. U. S. A. 110:E498-E506.

Zhou X, Ma H. 2008. Evolutionary history of histone demethylase families: distinct evolutionary patterns suggest functional divergence. BMC Evol. Biol. 8:294.

Zibetti C, Adamo A, Binda C, Forneris F, Toffolo E, Verpelli C, Ginelli E, Mattevi A, Sala C, Battaglioli E. 2010. Alternative splicing of the histone demethylase LSD1/KDM1 contributes to the modulation of neurite morphogenesis in the mammalian nervous system. J. Neurosci. 30:2521-2532. 


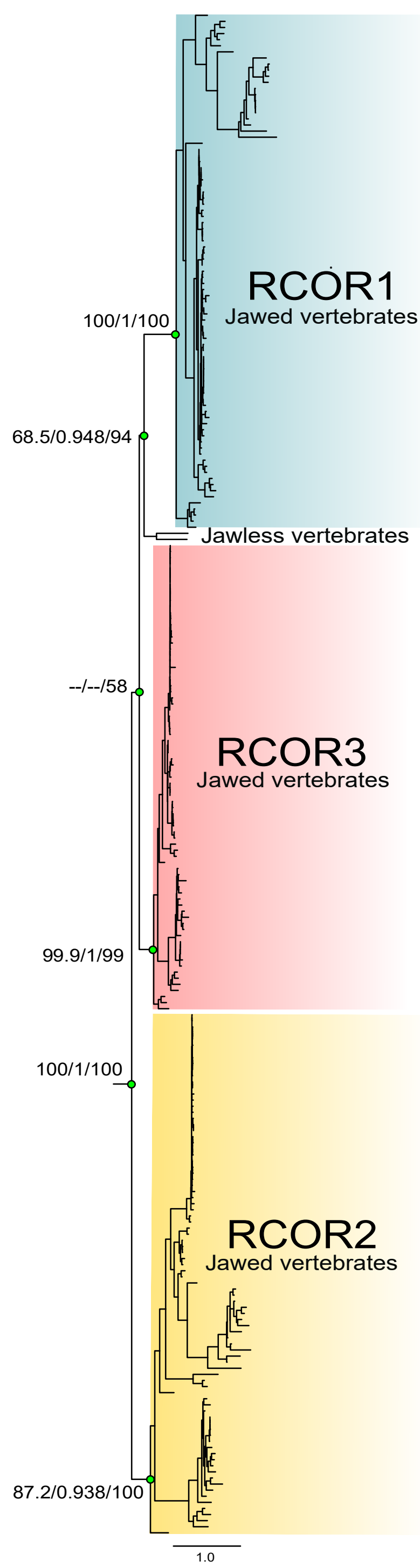

Figure 1: Maximum likelihood tree showing relationships among $R C O R$ genes of vertebrates. Numbers on the nodes correspond to support from the ShimodairaHasegawa approximate likelihood-ratio test, approximate Bayes test, and ultrafast bootstrap values. The scale denotes substitutions per site and colors represent gene lineages. Mitotic deacetylase-associated SANT domain protein (MIDEAS) sequences from human (Homo sapiens), gorilla (Gorilla gorilla), mouse (Mus musculus), and ferret (Mustela putorius furo) were used as outgroup (not shown). For more details of the species included in the analysis, refer to table $\mathrm{S} 1$. 


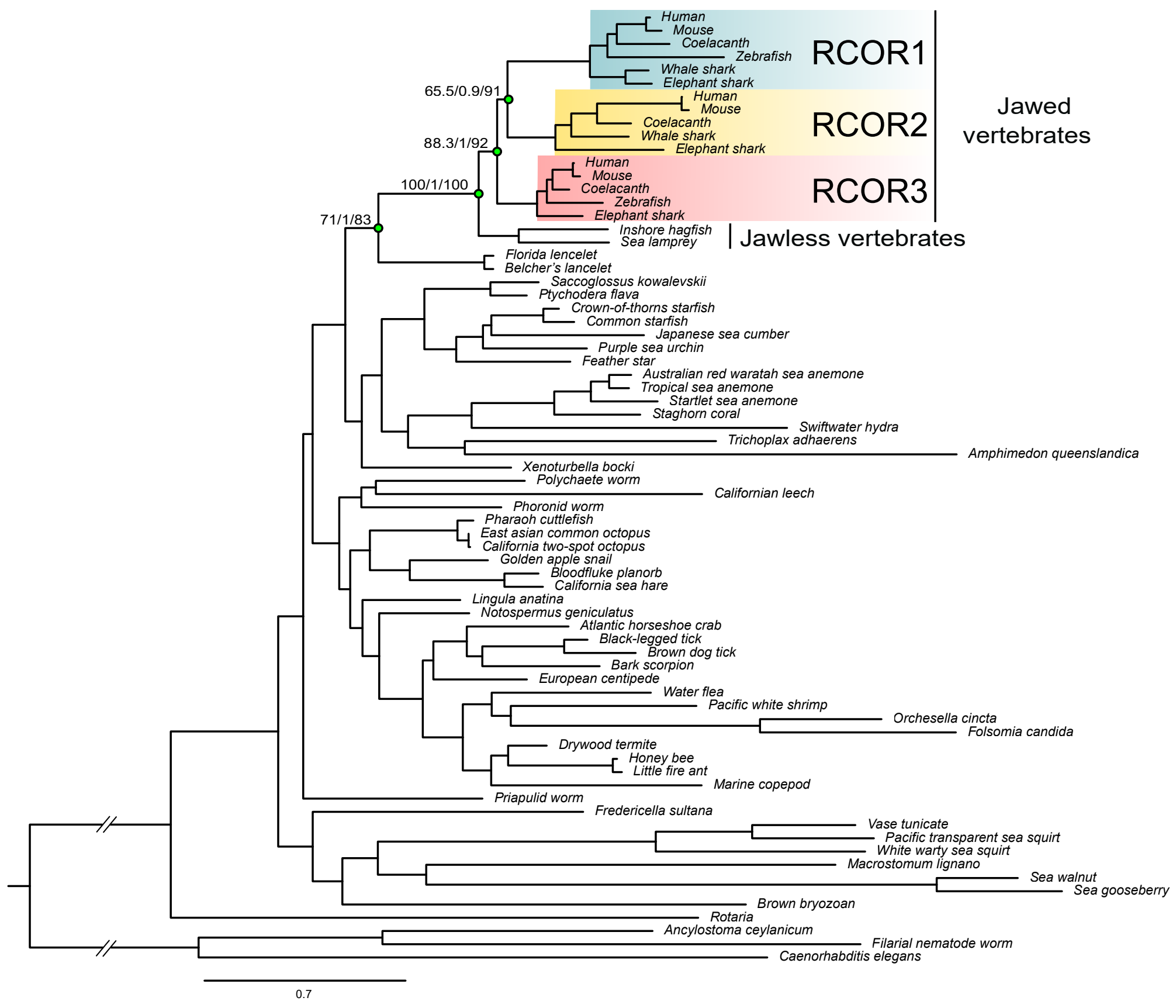

Figure 2: Maximum likelihood tree showing relationships among RCOR genes of metazoa. Numbers on the nodes correspond to support from the Shimodaira-Hasegawa approximate likelihood-ratio test, approximate Bayes test, and ultrafast bootstrap values. The scale denotes substitutions per site and colors represent gene lineages. Mitotic deacetylase-associated SANT domain protein (MIDEAS) sequences from human (Homo sapiens), gorilla (Gorilla gorilla), mouse (Mus musculus), and ferret (Mustela putorius furo) were used as outgroup (not shown). 


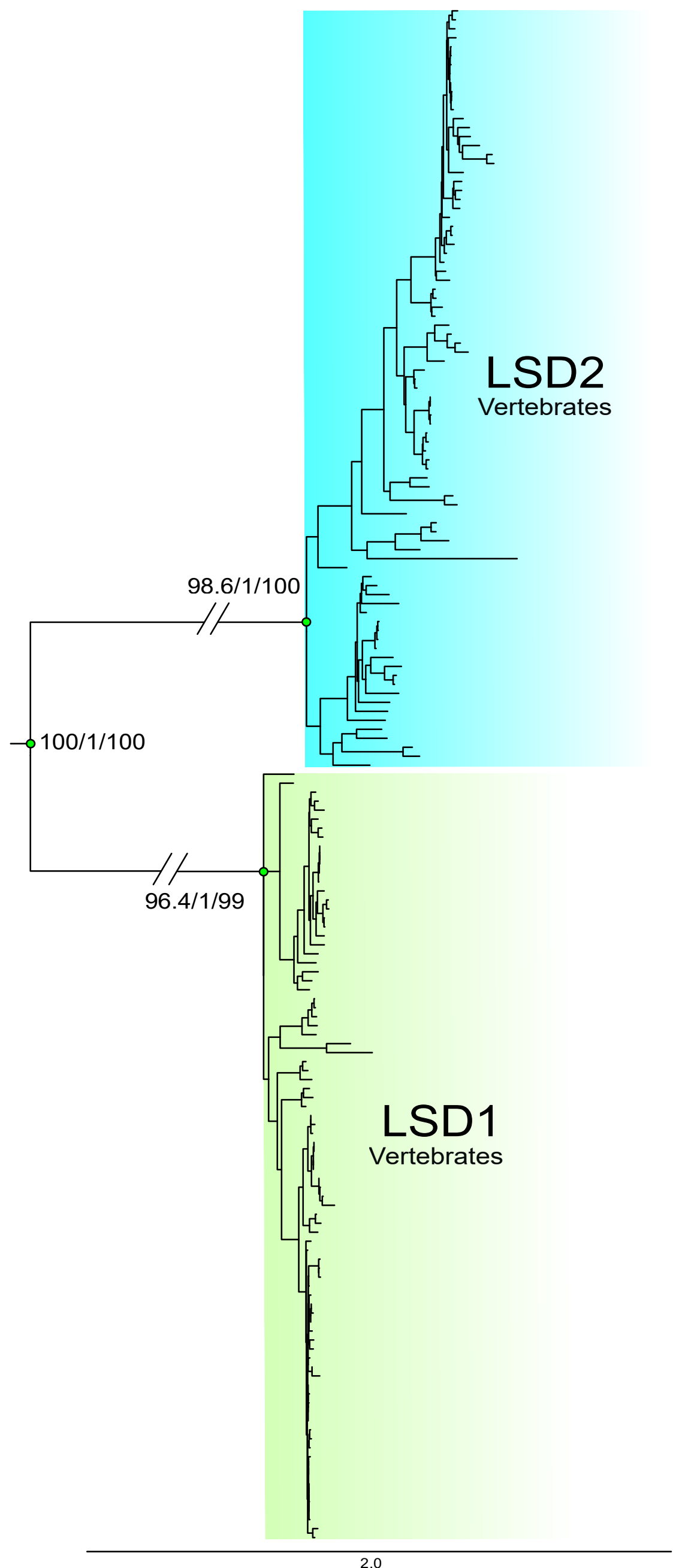

Figure 3: Maximum likelihood tree showing relationships among $L S D$ genes of vertebrates. Numbers on the nodes correspond to support from the ShimodairaHasegawa approximate likelihood-ratio test, approximate Bayes test, and ultrafast bootstrap values. The scale denotes substitutions per site and colors represent gene lineages. Monoamine-oxidases (MAO-A and MAO-B) sequences from human (Homo sapiens), chicken (Gallus gallus), spotted gar (Lepisosteus oculatus), and coelacanth (Latimeria chalumnae) were used as outgroup (not shown). 


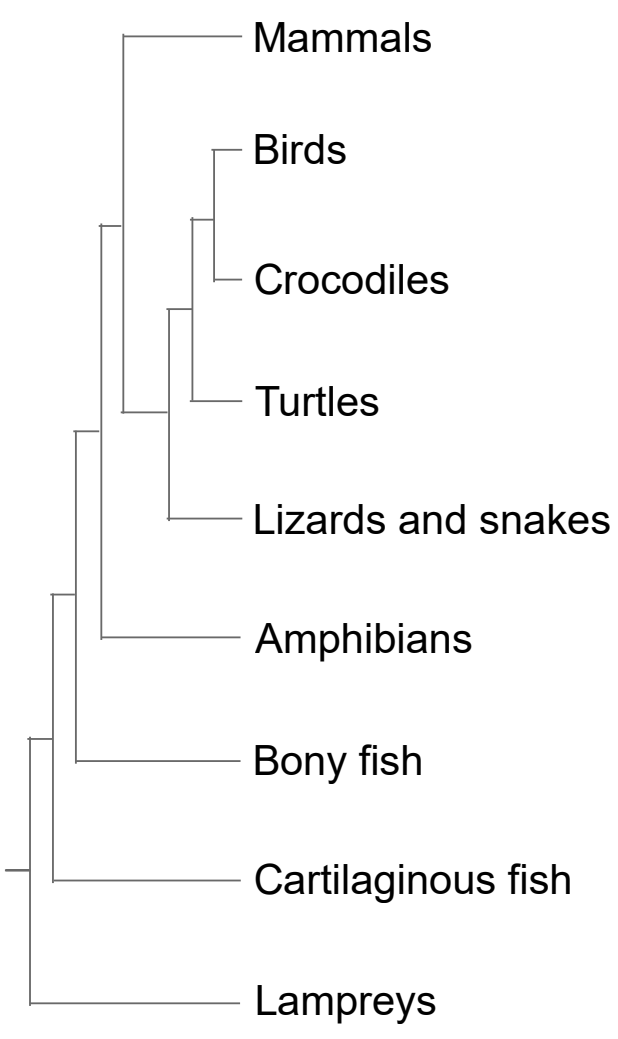

RCOR LSD

$1,2,3$

Microexon

Neuron-specific splicing regulatory sequences
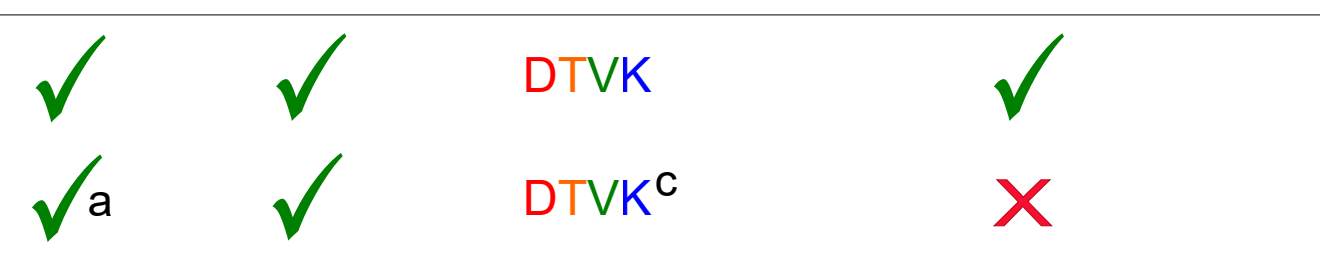

DTVK ${ }^{\mathrm{C}}$

$x$
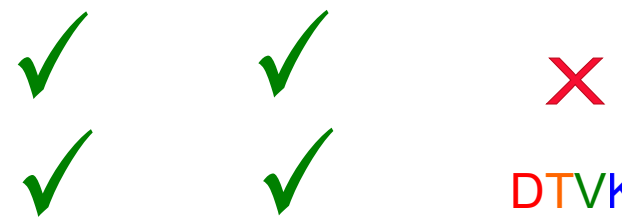

$\mathrm{X}$

DTVK
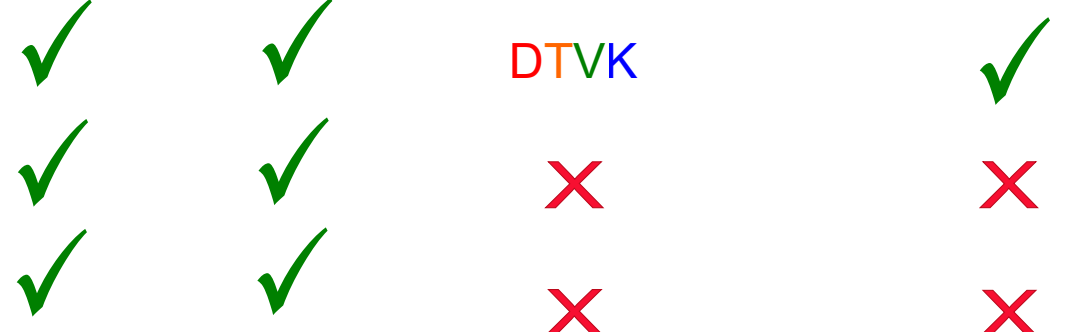

$x$

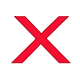

X

$x$

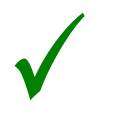

GERCTS

$x$

GERCTN

$x$

GERCTN

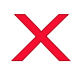

B
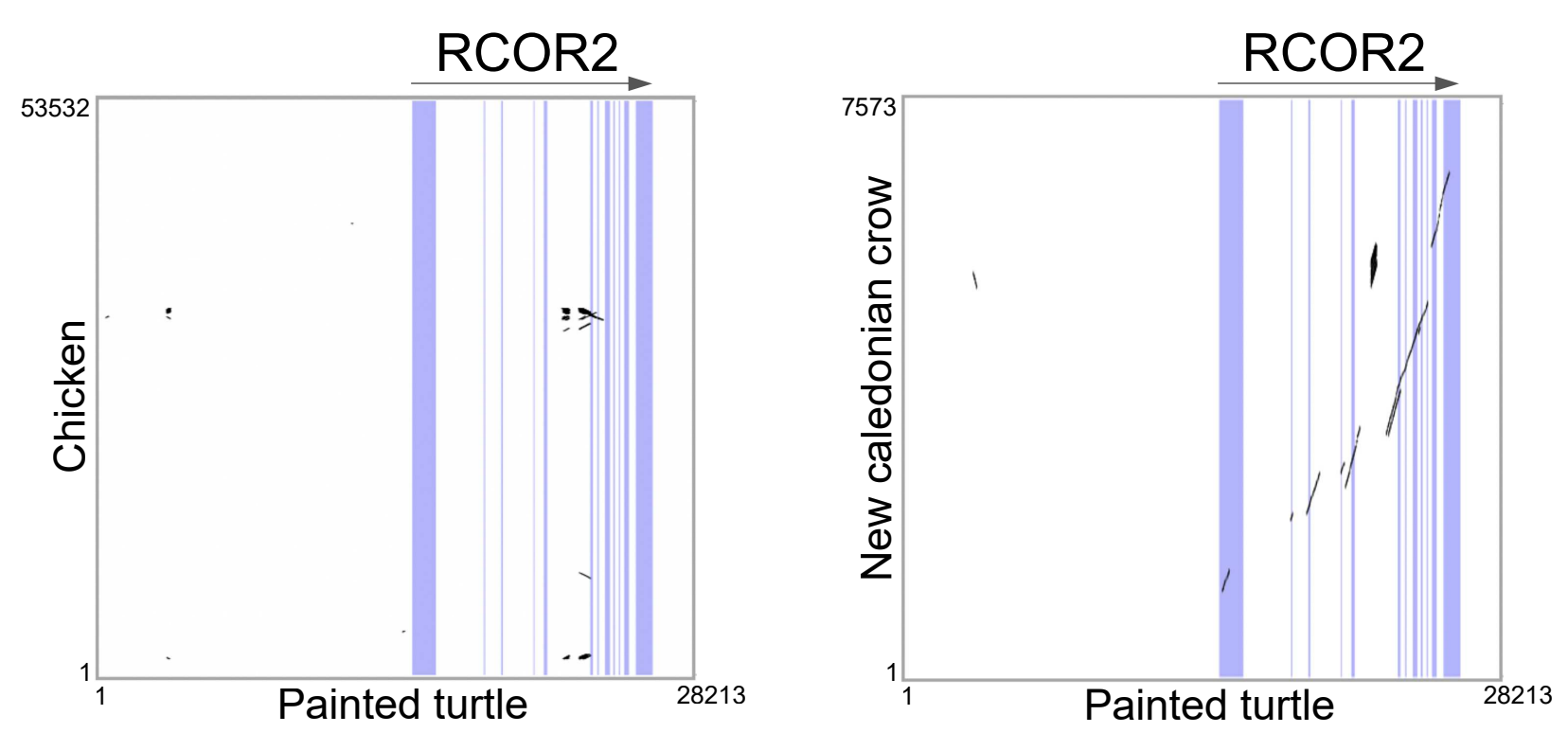

Figure 4: Phyletic distributions of RCOR and LSD genes, LSD1 microexon, and neuronspecific splicing regulatory sequences in vertebrates. A) Distribution of the RCOR and LSD genes, LSD1 microexon, and neuron-specific splicing regulatory sequences in main groups of vertebrates. B) Dot-plot of pairwise sequence similarity between the RCOR2 gene of the painted turtle (Chrysemys picta) and the corresponding syntenic region in the chicken (Gallus gallus) and New Caledonian crow (Corvus moneduloides). ${ }^{a}$ RCOR2 is present only in the bird orders Psittaciformes, Passeriformes, Accipitriformes, and Anseriformes. 'bampreys have a single copy of the RCOR gene. 'Some species of birds have a -DTVE- microexon. 


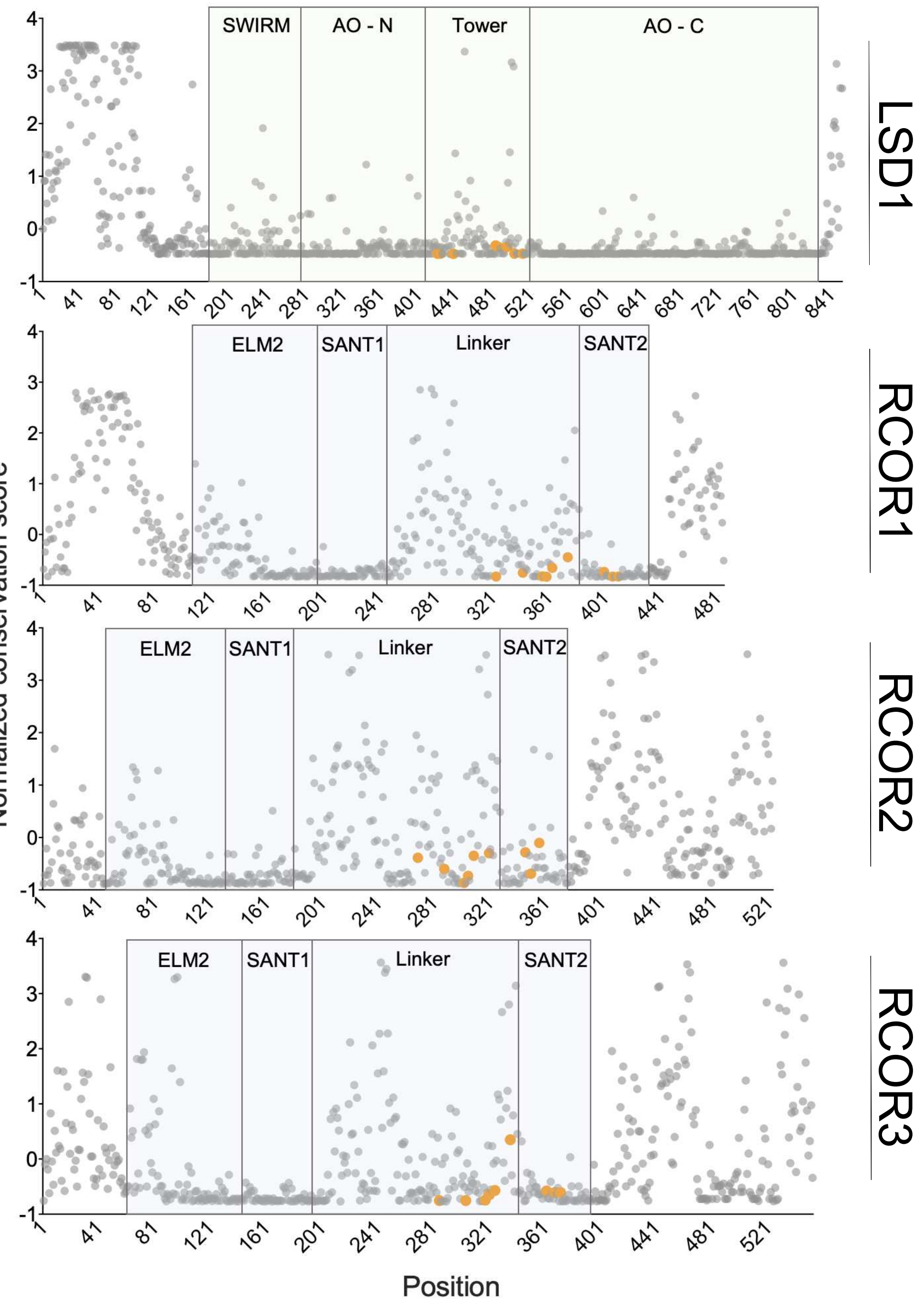

Figure 5: LSD1 and RCORs normalized conservation scores of jawed vertebrates. Orange dots represent the conservation scores of the key amino acids for the LSD1RCORs interaction ${ }^{44}$. 


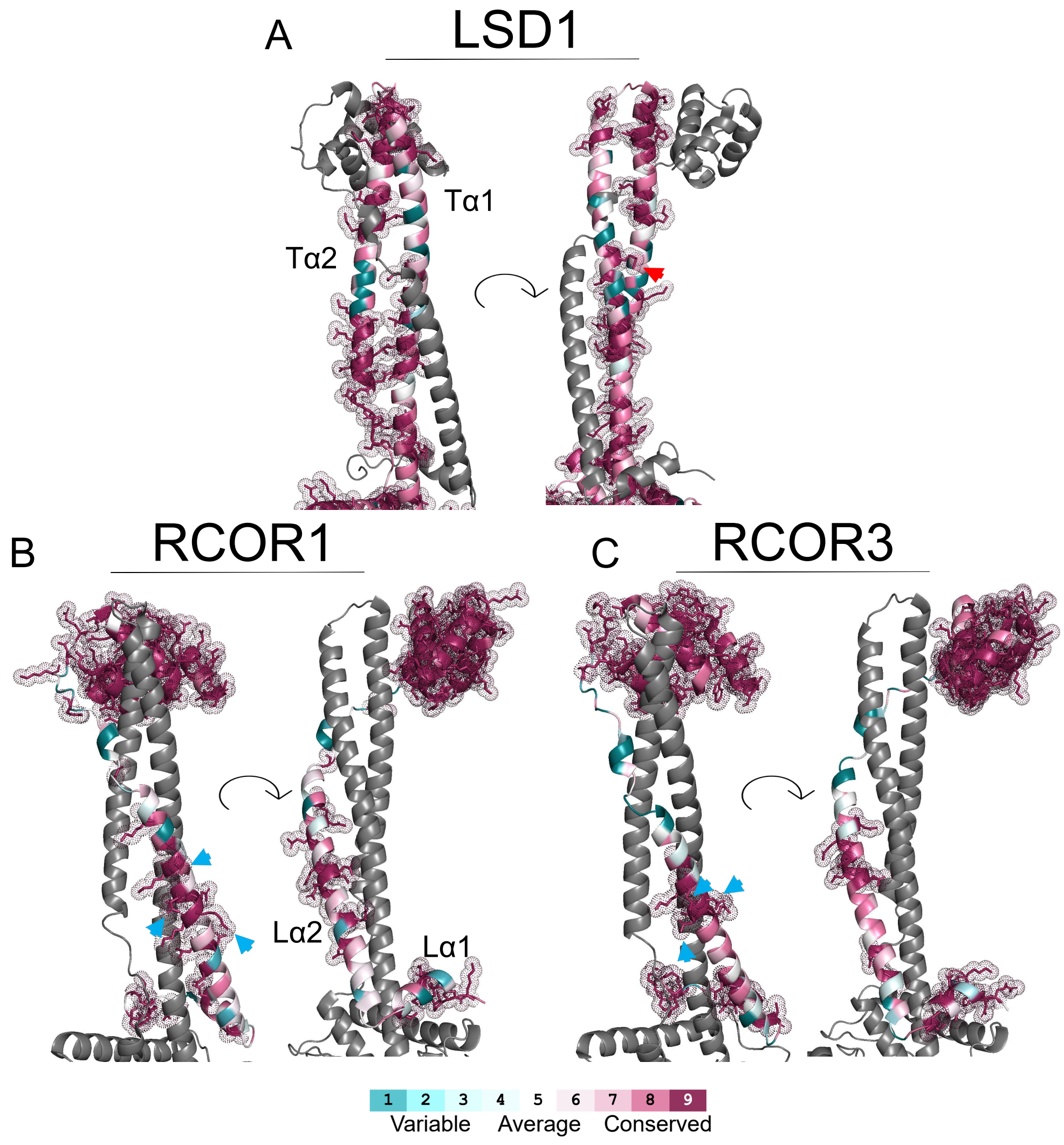

Figure 6: Color-coded 3D amino acid conservation of LSD1-RCOR protein complexes A) LSD1 tower domain residue conservation projected onto the structure of human LSD1RCOR1 (PDB: 2V1D). Red arrow shows Lys447 residue (see text). B) RCOR1 linkerSANT2 domain residue conservation projected onto the structure of human LSD1RCOR1 (PDB: 2V1D). Light blue arrows show Arg347, GIn350, and Gln354 residues (see text). C) RCOR3 linker-SANT2 domain residue conservation projected onto the structure of human LSD1-RCOR3 (PDB: 4CZZ). Light blue arrows show GIn315, Asn316, and Gln319 residues (see text). 
Animals

Human

Mouse

Fruit fly

\section{Fungi}

Fission yeast
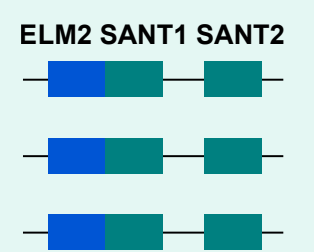

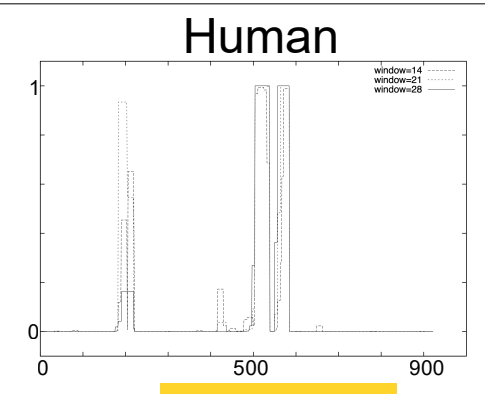

Entomophthora muscae
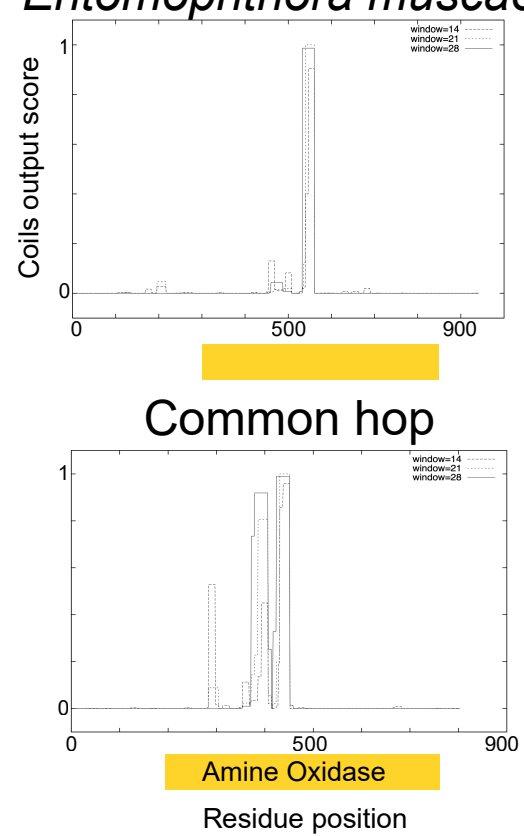

Residue position

Entomophthora muscae

Plants

Thale cress

Common hop
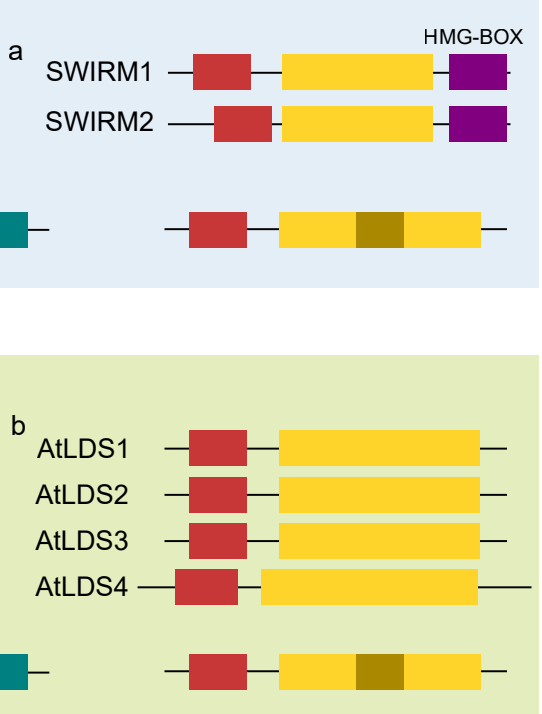

\section{Human LSD1 Tower domain}

Human LSD1 T S Y L S Q L D F N V N NKPVSLGQALEVVIQLQEKHVKDEQIEH WKKIVKTQEELK Entomophthora muscaeLSD1 A S Y L S H Q L D F N Y A G N N P V S L GQALE W I I K L Q E K A VK E K Q I Q H L TH L C E A Q K S I L Common hop LSD1 T S Y L S H T L D L Q V K G C P I S L G Q A L E WVI Q L Q E K S V K E K Q I E H L K L I V E L H DK LK Human LSD2 v S $\ldots \ldots \ldots \ldots$

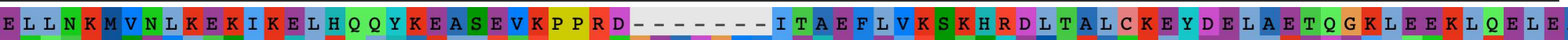

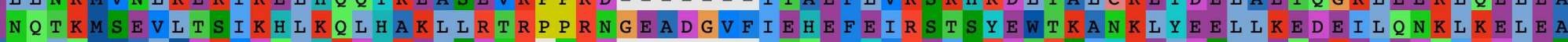
N Q L Q M M A L M DKVDQLH K DHKELSDNR-NRD- - - - - - I T QEFYFREISRDTRSIYKELDSLLEQEKEIEDQIRDLES

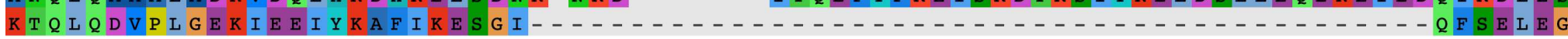

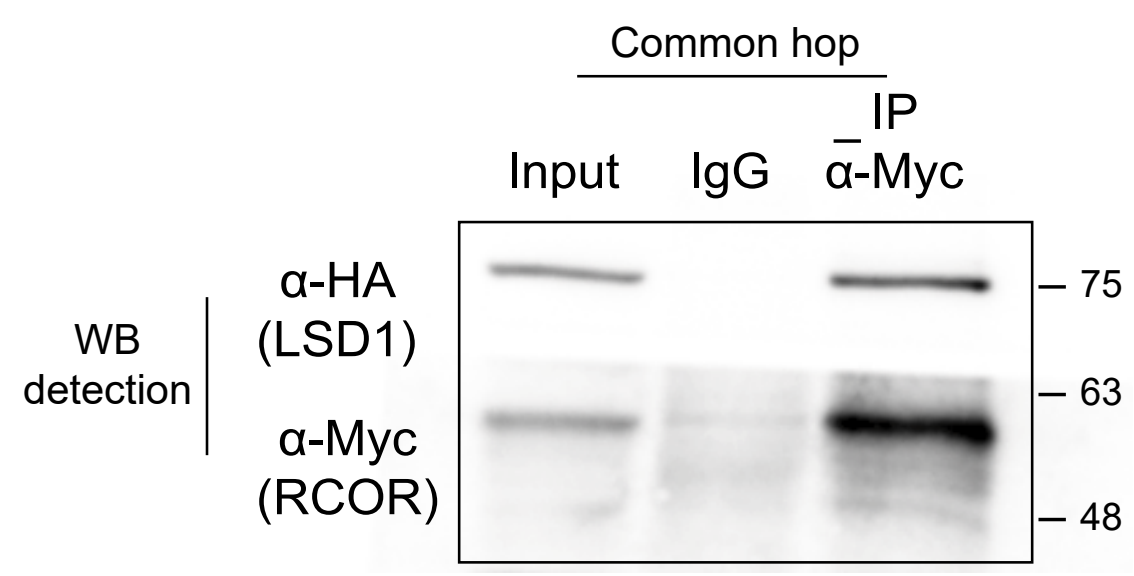

Figure 8: Molecular interaction between common hop LSD1 and RCOR proteins. A) (Left) Animal, fungi, and plant RCOR and LSD1 genes with their characteristic functional domains. The light brown section in LSD1's amino oxidase domain shows the prediction of a tower domain corresponding to an interruption in the amino oxidase domain and a strong prediction of a coiled-coil structure (Right). B) Multiple sequence alignment of human, Entomophthora muscae, common hop LSD1 and human LSD2 in the tower domain region of the human LSD1 sequence. C) Sodium dodecyl sulphate-polyacrylamide gel electrophoresis (SDS-PAGE) followed by immunoblot detection of the immunoprecipitate obtained using an $\alpha-M y c-R C O R$ antibody. Input represents whole-cell protein extracts, IgG serves as a negative control for the immunoprecipitation, while IP anti-Myc is the immunoprecipitation of common hop RCOR protein. Molecular weights are indicated at right. ${ }^{a}$ LSD fission yeast homologs according to Nicolas et al. $(2006)^{53},{ }^{b}$ LSD thale cress homologs according to Spedaletti et al. $(2008)^{54}$. 


\section{Supplementary Files}

This is a list of supplementary files associated with this preprint. Click to download.

- Supplementaryfigures.pdf

- Supplementarytables.xlsx

- Treessupportingdata.rar 